\title{
Integrating HIV care and HIV prevention: legal, policy and programmatic recommendations
}

\author{
Robert H. Remien ${ }^{a}$, Alan Berkman ${ }^{b}$, Landon Myerc, \\ Francisco I. Bastos ${ }^{d}$, Ashraf Kagee ${ }^{e}$ and Wafaa M. El-Sadr ${ }^{b, f}$
}

\begin{abstract}
Since the start of the HIV epidemic we have witnessed significant advances in our understanding of the impact of HIV disease worldwide. Furthermore, breakthroughs in treatment and the rapid expansion of HIV care and treatment programmes in heavily impacted countries over the past 5 years are potentially critical assets in a comprehensive approach to controlling the continued spread of HIV globally. A strategic approach to controlling the epidemic requires continued and comparable expansion and integration of care, treatment and prevention programmes. As every new infection involves transmission, whether vertically or horizontally, from a person living with HIV/ AIDS (PLWHA), the integration of HIV prevention into HIV care settings has the potential to prevent thousands of new infections, as well as to improve the lives of PLWHA. In this paper, we highlight how to better utilize opportunities created by the antiretroviral roll-out to achieve more effective prevention, particularly in sub-Saharan Africa. We offer specific recommendations for action in the domains of healthcare policy and practice in order better to utilize the advances in HIV treatment to advance HIV prevention.

(c) 2008 Wolters Kluwer Health | Lippincott Williams \& Wilkins
\end{abstract}

\section{AIDS 2008, 22 (suppl 2):S57-S65}

Keywords: healthcare policy, healthcare practice, integration of HIV prevention and HIV care, people living with HIV/AIDS

\section{Introduction}

Control of the global HIV/AIDS epidemic remains elusive as setbacks in some areas contrast with the gains made in others. On the positive side, HIV incidence and prevalence have declined in specific populations in a number of high seroprevalence countries in eastern and southern Africa, and the 2008 progress report of the World Health Organization (WHO) on the goal of universal access to HIV/AIDS treatment estimates that approximately 3000000 individuals in poor and middleincome countries were receiving antiretroviral therapy (ART) at the end of 2007 [1]. On the negative side, results from several prevention studies have been disappointing, including reports of a promising HIV vaccine candidate, two candidate microbicides and the use of the diaphragm for HIV prevention [2,3]. Male circumcision for HIVnegative men in high seroprevalence countries where the epidemic is driven by heterosexual transmission remains the only effective prevention strategy recently identified [4].

Although there is an urgent need for new and effective prevention technologies, it is imperative that effective interventions currently available be widely utilized if we are to slow the epidemic successfully now. An increased emphasis on expanded HIV testing in many areas of the globe is a positive step; however, the transformation of increased testing into a true strategic approach to controlling the epidemic requires continued and com-

${ }^{a}$ HIV Center for Clinical and Behavioral Studies, NY State Psychiatric Institute and Columbia University, New York, New York, USA; ${ }^{b}$ International Center for AIDS Care and Treatment Programs (ICAP) and Department of Epidemiology, Mailman School of Public Health, Columbia University, New York, New York, USA; ${ }^{\mathrm{C}}$ Infectious Diseases Epidemiology Unit, School of Public Health and Family Medicine, University of Cape Town, Cape Town, South Africa; ${ }^{d}$ Oswaldo Cruz Foundation - FIOCRUZ, Rio de Janeiro, Brazil; ${ }^{\circledR}$ Department of Psychology, Stellenbosch University, Cape Town, South Africa; and ${ }^{\mathrm{f}} \mathrm{Harlem}$ Hospital Center, New York, New York, USA.

Correspondence to Robert H. Remien, PhD, HIV Center for Clinical and Behavioral Studies, NY State Psychiatric Institute and Columbia University, New York, New York, USA.

Tel: +1 212543 5375; fax: +1 212543 6003; e-mail: rhr1@columbia.edu 
parable expansion of care, treatment and prevention programmes for all those identified with HIV. It is important that in the context of social justice, all populations have safe access to these programmes, including sexually active adolescents and those with minority status such as sex workers, men who have sex with men, substance users (including injection drug users; IDU) and undocumented immigrants and migrants. As every new HIV infection involves transmission from a person already infected, identifying individuals with HIV and enrolling and retaining them in a care system creates the opportunity to deliver biomedical and behavioral interventions that can potentially reduce both horizontal (person-to-person via sex or needles) and vertical (mother-to-child) transmission of HIV. According to the Joint United Nations Programme on HIV/AIDS (UNAIDS) global epidemic update for 2007, there were 420000 new infections in children that year, of which the vast majority was via mother-to-child transmission, with 2.1 million new infections in adults. The ratio of new children to adult infections is thus approximately $1: 5$, or $17 \%$ of all infections globally. The report also indicated that approximately $70 \%$ of new infections take place in sub-Saharan Africa, where the vast majority of HIV transmission is transmitted sexually among adults or from mother to child [4].

Salomon et al. [5], reporting on an epidemiological modelling exercise of the varying implementation of prevention and treatment programmes, argue for the need to fund and implement both fully in order to impact the HIV epidemic significantly over the next decade. To understand the potential synergies between care/treatment programmes and effective prevention, it is important to review some basic biological, epidemiological and behavioral concepts.

\section{HIV-RNA level in the blood is a strong predictor of HIV transmission}

This biological fact has been demonstrated both in the horizontal and vertical transmission of HIV [6]. West et al. [7] argue that strategic prevention programmes should target those most likely to transmit HIV, namely those with high HIV-RNA levels (i.e. primary/early HIV infection and late-stage disease). This is particularly important for HIV-serodiscordant couples, in whom the frequency of sexual encounters results in a high cumulative risk of HIV infection for the seronegative partner. Similarly, pregnant women with high viral loads are more likely to transmit HIV to their newborns.

\section{Co-morbid infections are associated with higher HIV-RNA levels and consequently an increased risk of HIV transmission}

Treating, or preferably, preventing co-morbid infections [tuberculosis, sexually transmitted infections (STI), opportunistic infections] may both minimize the associated morbidity and mortality for the HIV-infected individual and help lower the risk of transmission to others.

\section{Young people testing positive are a strategic group for effective strategies to decrease ongoing transmission}

In hyperendemic settings such as southern Africa, young women (15-24 years of age) who test positive in the antenatal clinics are likely to have primary or early HIV infection. They are therefore likely to have high viral loads and have most of their reproductive lives ahead of them. Enrolling these women into effective care can reduce the risk of mother-to-child transmission and improve their own health. Testing their male partners and bringing those who test positive into care can stabilize the family and potentially break the chain of transmission in their social network.

\section{Behavioral change interventions targeting HIV-positive individuals}

There are now several behavioral change interventions targeting HIV-positive individuals; however, they require time and repetition to be effective. Several sexual risk reduction interventions among PLWHA have been shown to be effective among adults and adolescents in high-resources settings [8-11]. These and many other behavioral interventions tend to have modest effects in the short term, but their impact may be maximized if delivered on multiple occasions over time.

The chronic care model increasingly adopted by HIV care/treatment programmes emphasizes the maintenance of patients in care over their lifetimes and offers opportunities to target behavioral and biomedical interventions repeatedly to a strategic population, individuals with HIV, their families and their partners. For example, Piwoz et al. [12] showed that increased exposure (over time) to an educational intervention for safer breastfeeding practices among pregnant women in Zimbabwe resulted in a concomitant reduction in the risk of late postnatal HIV transmission. A 15-session counselling intervention for HIV-positive adults that was integrated into clinic and community settings in the United States and was delivered over the span of a year demonstrated a significant reduction in unprotected sexual intercourse with HIV-negative or unknown status partners, with effects lasting until the 20 -month followup assessment [8]. This intervention was designed to help HIV-positive patients cope with distress and increase treatment adherence, as well as having placed an emphasis on the reduction of HIV transmission risk behaviors. It thus seems that important behavioral initiatives, such as support of disclosure of an HIV-positive status, partner and family testing, and improved adherence to ART and overall coping are all likely to benefit from consistent and repeated counselling. Psychosocial interventions ranging from opiate-substitution programmes to mental health programmes may help stabilize the lives of HIV-positive 
individuals, decrease the frequency of risk behaviors and improve their retention in care and adherence to treatment [13].

\section{Building on global successes}

The International AIDS Conference in Durban, South Africa in 2000 marked the beginning of serious efforts to address the devastating effects of HIV/AIDS on people living with HIV/AIDS (PLWHA) in resource-limited countries. These efforts were catalysed by grassroots mobilization focused on expanded treatment access. The success of the Brazilian national AIDS programme became both a model and an inspiration [14]. During the subsequent years, unprecedented energy, funding and planning were mobilized and have resulted in a substantial increase in access to ART in some of the poorest countries in the world.

This achievement is a potentially strategic asset in a comprehensive approach to controlling the epidemic. The challenge we address in this paper is how better to utilize opportunities created by the ART roll-out to achieve more effective prevention with PLWHA. We propose that the appropriate response to the statement that we cannot treat our way out of the HIV/AIDS epidemic is to use the advances in treatment to advance prevention efforts [5]. This strategy is partly based on the following observations.

Medical treatment for HIV has introduced a chronic care model of disease management with long-term patient contact rather than the acute episodic care model of healthcare that has dominated in almost all low and middle-income countries.

ART availability has necessitated improvements in the logistical systems needed for the planning, purchasing and distribution of medications and commodities required by care and treatment programmes. These same systems can be used to supply currently available HIV/STI prevention technologies (e.g. male and female condoms) as well as non-barrier contraception that allow HIV-affected families to plan their children, and could be used for the distribution of future interventions if shown to be effective in reducing HIV transmission.

Linkage of HIV care and treatment programmes with antenatal clinics, obstetric services, and maternal and child health programmes more generally has been achieved in some settings. Connecting rapidly expanding HIV care and treatment services with existing prevention of mother-to-child transmission (PMTCT) programmes has the potential to improve coverage and ultimately reverse the public health shortcomings of stand-alone PMTCT programmes throughout much of sub-Saharan
Africa [15]. These linkages can serve as models of coordinated care that may enhance both treatment and HIV prevention efforts.

Knowledge of HIV seropositivity has been shown in some studies to be associated with the adoption of less risky behaviors [16,17]. HIV voluntary testing and counselling initiatives have been shown to be costeffective in different low and middle-income countries, as demonstrated by empirical studies carried out in Kenya and Tanzania [18] or by a recent meta-analysis [19]. The meta-analysis by Denison et al. [19] showed that individuals who received voluntary testing and counselling were less likely to engage in risky behaviors (compared with controls), although the effect was found to be modest with a lack of significant impact in terms of a reduction in the number of sexual partners. Diagnosing HIV and providing access to treatment, counselling and supportive services may thus contribute substantially to helping curb the HIV epidemic. In addition, knowledge of HIV seropositivity and the subsequent receipt of HIV treatment may also extend the lives of HIV-infected individuals. The latter effect can extend beyond the individual in the context of HIV-infected parents, leading to stabilization of the lives of their children and mitigating the vulnerability that accompanies orphaning.

The potential for direct integration of HIV prevention into HIV care and treatment programmes includes, for horizontal transmission: behavioral interventions and provision of methods for prevention for PLWHA; intensive counselling on sexual risk reduction for HIVserodiscordant couples, including for those wishing to have children; effective ART, treatment of STI and opportunistic infections to decrease HIV-RNA levels and reduce the risk of transmission through unprotected sex or needle sharing. For vertical transmission: the provision of effective family planning for HIV-infected individuals who do not wish to have children; early identification of pregnancy among HIV-infected women enrolled in care programmes allowing the use of optimal antiretroviral regimens and ensuring effective intervention to prevent intrauterine, peripartum and early postpartum HIV transmission. The latter includes counselling for exclusive breast-feeding or other safer approaches to infant feeding.

\section{Overcoming obstacles to effective coordination of HIV prevention and HIV care}

Failure to retain patients in care, and for those on ART failure to help them achieve high levels of treatment adherence have negative consequences for the individual in terms of disease progression and for public health in terms of the development and transmission of 
drug-resistant strains of the virus [20-22]. In addition, some have raised concern that the availability of effective HIV treatment may lead to a diminished perception of negative consequences of HIV infection and an increased complacency towards safer sex practices [23-26]. As the number of people on ARTworldwide increases, so do the opportunities for the emergence of viral resistance. This reality should not be considered a barrier to increased roll-out, but rather underscores the need for the integration of safer sex counselling as well as adherence counselling into ART delivery programmes.

There are many structural and contextual barriers impeding the implementation of effective, coordinated prevention and care programmes for PLWHA, including the long-standing practice of separating prevention and treatment. In the US model, for example, prevention is the domain of public health and treatment is provided through the medical care system. In resource-constrained countries, a similar model largely exists and, thus, it is imperative for an innovative public health approach to combine the two and build on the existing strengths of each of these programmes.

There are numerous other obstacles that have shaped and sometimes distorted the response to HIV/AIDS in different countries, covering a wide range of domains. In this paper, we focus on the most salient among these and offer recommendations for integrating HIV prevention into HIV care and treatment programmes for PLWHA worldwide.

\section{Stigma, discrimination and associated legal and policy impediments}

In many contexts, taboos associated with discussing sex, homosexuality, condoms and ART persist. These are deemed 'foreign' and considerable stigma is associated with being HIV infected. If patients feel a need to conceal their HIV-positive status within their social network, they may engage in efforts to hide their medications, change dosing schedules, or suppress observable side effects of treatment, all of which may have implications for adherence [27]. Similarly, they may be reluctant to use condoms during sex for fear it may be interpreted by partners that they are HIV positive, thus 'outing' their status.

The lack of political will and ideologically motivated rather than evidence-based policy has impeded effective prevention and treatment efforts in some settings. For example, the federal ban on needle exchange programmes in the United States and the prohibition of the delivery of methadone to opiate-dependent individuals by the Russian government have driven vulnerable populations away from appropriate interventions and have contrib- uted to HIV transmission rates [28,29]. Similarly, discriminatory policies such as the criminalization in many countries of sex work, same-sex sexual activity and engaging in unprotected sex without the disclosure of an HIV-positive status is likely to impede HIV prevention and treatment programmes [30,31]. More importantly, these taboos impede communication between patients and healthcare providers about sex and thus impede the discussion of sexual risk reduction strategies. If patients are unable to discuss these issues comfortably with healthcare workers, they may abandon care rather than confront the disapproval of providers and counsellors.

\section{Sexual and reproductive health}

For many HIV-infected individuals, pregnancy desire and intent is a motivating factor for engaging in unprotected sex, despite the presence of HIV [32]. There is growing evidence to suggest that HIV infection modifies, but does not remove, individuals' desires to have children [33]. Although most attention to fertility desires among HIVinfected individuals has focused on women, there is mounting evidence to suggest both that HIV-infected men often desire children [34] and that they have a substantial influence over their female partners' fertilityrelated behaviors. Efforts to address fertility-related issues among HIV-infected individuals must thus target women and men alike. This is important as reproductive desires have an impact on both the horizontal as well as the vertical transmission of HIV.

Given the disproportionate impact of HIV on the reproductive lives and health of women, the failure to link HIV treatment programmes to reproductive health services contributes to the disproportionate negative impact of HIV on women's health [32]. Basic components of primary healthcare, most notably access to effective forms of contraception, are often not provided as part of routine HIV care and treatment. The synergy between reproductive healthcare and HIV prevention activities has been slow to materialize on the ground. In light of evidence that preventing unwanted pregnancies among HIV-infected women may be the most cost-effective form of PMTCT [35], the failure to integrate reproductive healthcare services to HIV care and treatment effectively represents a substantial missed opportunity in HIV prevention [36].

In most countries, even PMTCT programmes, which come closest to linking HIV and reproductive healthcare services, struggle to bridge this divide and have been traditionally conceptualized as a stand-alone prevention intervention located within maternal and child health services. There are conceptual, historical and operational reasons for this situation, and these may contribute to the limited coverage of PMTCT programmes in many 
sub-Saharan African countries. The integration of PMTCT with HIV care and treatment is likely to achieve clear positive outcomes for both prevention and treatment efforts.

\section{A fragile healthcare workforce and infrastructure}

The scale-up of ART programmes has challenged an already overstretched healthcare workforce in most settings and, thus, attempts to provide more intensive HIV prevention support will necessarily add to this burden. In the United States, a study demonstrated that in most HIV treatment and care settings there was no routine provision of safer sex counselling directed at the very people who are able to transmit HIV to their uninfected partners [37].

An increasing emphasis in resource-limited settings has focused on 'task-shifting' in an effort to ensure that specific tasks needed in the framework of HIV care and treatment are accomplished while initiating longer-term efforts to establish new cadres of workers and retain all levels of healthcare workers. Lay or peer counsellors are an important cadre of workers who provide important programmatic activities such as HIV counselling, adherence support and community outreach. Relatively few systems have formal programmes of behavioral prevention for positive individuals, and an effort to bring such programmes to scale will undoubtedly also demand the involvement of lay/peer counsellors. Whereas the deployment of lay counsellors is laudable, it is important that in the context of competing demands on resources, adequate emphasis be placed on the appropriate training and supervision of these workers. Much of the counselling currently provided by lay personnel is not based on any formal behavioral change theory and is largely limited to the dissemination of information rather than processing of patients' emotions and cognitions to alleviate distress and alter long-standing behavior patterns. In the absence of adequate supervision, training and support for counsellors, the possibility of null or iatrogenic effects exists [38].

Behavior change, whether directed towards HIV prevention or retention and adherence to care, may not lend itself to the strict algorithmic approach that has made it possible to expand HIV treatment programmes rapidly. The challenge therefore remains for behavioral scientists to develop, test and disseminate innovative methods to incorporate theory-based effective interventions into public health practice. Correspondingly, healthcare planners must be educated about the vital need for behavior change interventions if the espoused goals of universal access to care, treatment and prevention are to be met.

\section{Lack of mental health and substance use treatment}

Adults and children living with HIV, and those at risk of acquiring HIV, have elevated rates of psychological distress and psychiatric co-morbid conditions, especially mood and anxiety disorders (including posttraumatic stress disorder), and substance use disorders [39-41]. This psychiatric co-morbidity in the context of HIV can contribute to increased substance use and sexual risk behavior, poor treatment adherence, interpersonal violence and other maladaptive behaviors, as well as more rapid disease progression and diminished health outcomes [42-45]. Substance abuse can contribute to poor immune functioning and disease progression among PLWHA. Among other problems, alcohol use can modify liver drug metabolism, thus complicating treatment for patients with $\mathrm{HIV} /$ hepatitis C virus co-infection, as alcohol may compromise pegylated interferon therapy and favor liver disease progression [46]. Similarly, mood disorders, particularly depression, have been associated with HIV disease progression and poor quality of life among PLWHA [47,48].

It has become increasingly evident that mental health vulnerabilities 'travel together' and contribute to ongoing HIV transmission in many regions of the world. As recently noted by Sweat and colleagues [35], the provision of psychosocial services for PLWHA pales in comparison with the growing needs of PLWHA living in impoverished and underserved communities. The high prevalence of co-morbid medical and psychiatric conditions among PLWHA highlight the pressing need to co-locate different services and specialists to provide comprehensive care for individuals with special needs and overlapping medical and psychological conditions.

The unavailability of safe and easy access to needle and syringe exchange programmes and safe injecting environments for IDU contributes to the ongoing transmission of HIV in this population as well as the continued spread of HIV through sex within their social and sexual networks $[49,50]$. Several studies have shown that once HIV-positive substance users have access to the necessary support, they are able to adhere to antiretroviral regimens and thus experience treatment benefits despite the complex circumstances involved in their treatment $[51,52]$.

With the expansion of HIV treatment programmes in resource-limited countries, there is an urgent need to formulate and test innovative management and preventive strategies among HIV-infected individuals with mental health and substance abuse problems in order to minimize loss to follow-up, improve adherence to ART and help individuals and their partners live healthier and safer lives, as well as adopting behaviors to minimize the risk of HIV transmission to others. The provision of ART without 
accompanying mental health and substance use services for those who need it hampers achieving lasting beneficial impact on the HIV epidemic.

\section{Recommendations}

Coordination of care, treatment and prevention services at the planning and operational levels

Donors and governments should be encouraged and assisted to integrate appropriate aspects of HIV prevention with HIV care and treatment more effectively. Ultimately, there is a need to expand the types of services delivered to HIV-infected individuals on the ground. Systems and operational research are critically important to understanding how this can be achieved in diverse countries based on the characteristics of their healthcare services. Although the goal of coordinated HIV services at the community level is the same across countries, each country's planners will need to assess existing health systems and the burden and characteristics of their nation's epidemic to develop an appropriate blend of linkages, co-location or totally integrated services. Donor funding should encourage flexibility and nurture the establishment of creative models of integrated programmes. As an example, Brazil's long-term commitment to the integrated delivery of prevention and care to people living HIV/AIDS has been documented [14,53,54], and has demonstrated how the implementation of comprehensive prevention strategies can be successfully accomplished in a middle-income country. Furthermore, a wellintegrated and far-reaching system of monitoring and evaluation has recently been established in the country [54], paving the way for similar initiatives in other middle and hopefully low-income countries. This type of approach is compatible with the recommendation made by the Institute of Medicine regarding focussing on integrated prevention and treatment efforts and support for behavioral prevention efforts [55].

\section{Development of programmes focused on women's needs that integrate reproductive health and HIV services through co-location and/or effective linkages}

Across countries, women typically come into contact more often with the healthcare system and may serve to help other family members access healthcare services. Orienting services to the particular needs of women, which include care of their partners and children when appropriate, is a strategic way to improve adherence to care and treatment programmes. Because of the disproportionate impact of the HIV epidemic on women (in both social and biomedical terms), the separation of prevention and care/treatment services is likely to exacerbate the negative consequences of HIV disease for women. In particular, the triad of HIV care/ treatment, PMTCT and reproductive healthcare, should be coordinated at the policy, planning and operational levels as a seamless continuum. Integrated services would provide opportunities to deliver interventions across the spectrum of needs of HIV-infected women.

Development of guidelines for safer reproductive strategies for HIV-infected women and men with training of healthcare workers on the sexual and reproductive rights of the patient

In order to better support informed and appropriate sexual partnering and reproductive choices among HIV-positive individuals and facilitate effective prevention interventions within care and treatment programmes, interventions are required at two discrete points. First, at a policy level, clear and explicit statements and effort are required to underscore the importance of free and informed sexual and reproductive choices among HIV-infected women and men (as among all individuals). For individuals considering whether to have a child, clear messages are required regarding the potential risks involved (including vertical transmission of HIV to the child, and possibly horizontal HIV transmission in the case of serodiscordant partnerships), as well as the magnitude of these risks relative to that of a safe pregnancy. Second, policies that support informed choice must be supported by services that allow individual choices to be enacted as safely as possible. Individuals who have decided to have a child require information regarding safe conception and childbearing; individuals who decide not to have a child require access to effective contraception, along with information on sexual risk reduction. Specific interventions for healthcare providers are needed as they often act as the principle 'gatekeepers' to health-related information. Healthcare providers (including nurses, doctors and counsellors) require relevant biomedical information on fertility and HIV infection. Many providers may benefit from values clarification interventions, which seek to distinguish between providers' professional responsibilities and their personal beliefs. Also, standards of care guidelines are needed for routine sexual behavior assessments for all people living with HIV, in conjunction with HIV care and treatment, as well as routine screening for and treatment of all STI.

\section{Human right advocacy for changes in policy and law and their implementation to ensure access to services for all HIV-infected individuals, including high-risk marginalized populations}

As international human rights law prohibits discrimination on the basis of HIV status, all countries must ensure that no laws, policies or practices discriminate in access to prevention, care, treatment as well as reproductive and sexual health information and services on the grounds of HIV status, as well as race, color, sex, or national or social origin [56]. Although law reform is a time-consuming political process requiring broad consensus among policy makers, law enforcement practitioners, healthcare professionals and communities, there are specific policies that can be implemented to improve public health in terms of HIV. Safe access is needed to 
prevention strategies including sexual and reproductive health and HIV-related services for all people including adolescents and vulnerable populations such as sex workers, men who have sex with men, substance users (including IDU), and undocumented immigrants and migrants. The availability of confidential youth-friendly health services for adolescents without legal and policy constraints is critical. In addition, programmes are also needed to address the needs of minority and marginalized populations with HIV.

\section{'Scale-up' of effective behavioral interventions and training of staff to provide these interventions}

Various sexual risk-reduction behavioral interventions have been shown to be effective among HIV-infected men, women and adolescents [8,57-59], although these studies have been conducted primarily in the United States and have not evaluated their effect on HIV transmission or other biological outcomes. More research is thus needed that evaluates the effectiveness of various interventions on HIV acquisition as well as studies of effectiveness of the behavioral interventions that have been shown to be effective in the United States in resource-limited countries. Even with limited data on the effectiveness of the behavioral interventions on HIV transmission and lack of data on their impact when implemented in a programmatic setting, offering prevention services to HIV-infected individuals is recommended as an important approach to the control of the HIV epidemic in the United States [60]. Both the Institute of Medicine and the Centers for Disease Control and Prevention have advocated the inclusion of 'prevention with positives' programmes in a coordinated national prevention strategy $[55,61]$.

The development and dissemination of innovative tools is needed in order to assist in their use by a broad range of counsellors and healthcare providers. For example, multimedia technology, such as the use of laptops and pre-programmed psycho-educational materials, as well as standardized training and tools have been used effectively in other health-related interventions in resource-poor settings, showing improvements in health behaviors among patients and enhanced cost-effectiveness when implemented by community health workers [62-65]. Such tools may have the advantage of: (i) the use of videos and pictures to teach memorable lessons to patients, including those with poor literacy, about HIV care, adherence and sexual practices; (ii) improving quality control and consistency of delivery of intervention content; (iii) and necessitating limited supervision and training.

\section{Provision of mental health and substance use services to HIV-infected individuals who need it} It is important to find ways to provide and sustain much needed mental health services for all individuals and families living with HIV, both in resource-poor countries with limited mental health infrastructure, as well as in settings that already have the capacity and means. HIV treatment guidelines should include routine screening for mental illness as part of regular assessments and the provision of services for individuals with such conditions, including appropriate medications and counselling interventions. Creative approaches are needed for the provision of mental health services in settings in which such services may be perceived as unfamiliar to the culture and the local capacity of the healthcare system. For example, psychological support, particularly around issues such as disclosure, adherence and sex can be provided by peer counsellors, trained nursing staff and lay counsellors from the community. At other times we may need to build more formal mental health infrastructure (i.e. for the treatment of psychiatric disorders). Innovative partnerships between community-based organizations and clinical facilities can assist in enhancing available resources. Whereas it may be difficult for some to justify the provision of mental health or other psychosocial services when there are pressing physical needs (e.g. physical symptoms, food, shelter), to ignore the former may severely limit the ability to control the transmission of HIV and to enable individuals and families to remain engaged in treatment programmes. Similarly, as a result of its direct link to HIV transmission, there is a specific need to implement and sustain needle and syringe exchange programmes as HIV prevention strategies, as well as ensuring the availability of pharmacological and behavioral treatments for PLWHA coping with substance use disorders.

\section{Building on the lessons of the antiretroviral therapy scale-up ' $3 \times 5$ '}

The rapid expansion of HIV treatment programmes over the past 5 years has important lessons for all sectors of the global AIDS community. It has demonstrated that dramatic advances can be achieved by setting clear goals and numerical targets, by increased funding, and by exceptionally hard work by a broad coalition of stakeholders. It has also clarified the need for a global commitment to strengthening weak healthcare systems across the world. The evaluation of $3 \times 5$ done by the WHO, as well as the evaluation of the President's Emergency Plan for AIDS Relief (PEPFAR) carried out by the US Institute of Medicine, should be disseminated and discussed as the HIV community plans for the International AIDS Society Conference in Mexico City in 2008. If commitments to universal access by 2010 are to be achieved, they must build on a critical assessment of successes and shortfalls of current efforts. This must include a discussion of how access to treatment can advance prevention endeavors. It is the responsibility of the global AIDS community, professionals, PLWHA and advocates, to develop a plan of action with clear timelines for the achievement of an integrated approach 
to HIV prevention linked to care and treatment. Whereas the demand made by activists at the Durban AIDS Conference in 2000 that effective HIV treatment be made available to every person who needs it was dismissed by many as utopian, it may now, in retrospect, be seen as visionary. The progress since that meeting has clarified the obstacles that must be overcome over the next decade at the international, governmental and community levels in order to fulfill this vision. If this momentum continues, it should serve as a catalyst for a new and more comprehensive approach that aims at control of the spread of HIV through effective integration of HIV prevention with care and treatment. There is a need to learn the lessons from the past, renew and broaden the current vision, and set future concrete targets that link prevention with treatment efforts. As WHO Director-General Margaret Chan has said 'What gets measured, gets done'.

\section{Acknowledgments}

The authors would like to thank all of the participants in the 2007 UCLA Social Justice, Human Rights, and HIV Prevention Think Tank meeting in Sydney, Australia, especially Helen Rees and Judy Auerbach for serving as discussants and for their thoughtful comments on an early draft of this manuscript. This work was generously supported by the Ford Foundation. Dr Remien was also supported by the HIV Center for Clinical and Behavioral Studies (P30-MH43520) at the New York State Psychiatric Institute and Columbia University. Dr Kagee was supported by the Columbia University-Southern African Fogarty AIDS International Training and Research Program (D43-TW00231). Finally, we would like to acknowledge the insights from many colleagues and PLWHA from around the world.

Conflicts of interest: None.

\section{References}

1. WHO, UNAIDS and UNICEF. Towards universal access: scaling up priority HIV/AIDS interventions in the health sector. Progress report, June 2008. Geneva, World Health Organization, 2007 (http://www.who.int/hiv/mediacentre/2008progressreport/en/ index.html, accessed 19 June 2008).

2. US National Library of Medicine. National Institute of Health. Clinical alert: immunizations are discontinued in two HIV vaccine trials. 24 September 2007. Available at: http://www. nlm.nih.gov/databases/alerts/hiv_step_study.html. Accessed: 27 September 2007

3. Padian NS, van der Straten A, Ramjee G, Chipato T, de Bruyn G, Blanchard $K$, et al. Diaphragm and lubricant gel for prevention of HIV acquisition in southern African women: a randomised controlled trial. Lancet 2007; 370:251-261.

4. Joint United Nations Programme on HIV/AIDS. AIDS epidemic update, December 2007. UNAIDS/07.27E/JC1322E. Geneva: UNAIDS, 2007.

5. Salomon JA, Hogan DR, Stover I, Stanecki KA, Walker N, Ghys $\mathrm{PD}$, et al. Integrating HIV prevention and treatment: from slogans to impact. PLOS Med 2005; 2:50-56.
6. Wawer MJ, Gray RH, Sewankambo NK, Serwadda D, Li X, Laeyendecker $\mathrm{O}$, et al. Rates of HIV-1 transmission per coital act, by stage of HIV-1 infection, in Rakai, Uganda. I Infect Dis 2005; 191:1403-1409.

7. West GR, Corneli AL, Best K, Kurkjian KM, Cates W Jr. Focusing HIV prevention on those most likely to transmit the virus. AIDS Educ Prev 2007; 19:275-288.

8. The NIMH Healthy Living Project Team. Effects of a behavioral intervention to reduce risk of transmission among people living with HIV: the healthy living project randomized controlled study. J Acquir Immune Defic Syndr 2007; 44:213-221.

9. Kalichman SC, Rompa D, Cage M, DiFonzo K, Simpson D, Austin J, et al. Effectiveness of an intervention to reduce HIV transmission risks in HIV-positive people. Am J Prev Med 2001; 21:84-92.

10. Richardson JL, Milam J, McCutchan A, Stoyanoff S, Bolan R, Weiss J, et al. Effect of brief safer-sex counseling by medical providers to HIV-1 seropositive patients: a multi-clinic assessment. AIDS 2004; 18:1179-1186.

11. Rotheram-Borus MJ, Lee MB, Murphy DA, Futterman D, Duan $\mathrm{N}$, Birnbaum JM, et al. Efficacy of a preventive intervention for youth living with HIV. Am J Public Health 2001; 91:400-405.

12. Piwoz EG, Humphrey JH, Tavengwa NV, Iliff PJ, Marinda ET, Zunguza $C D$, et al. The impact of safer breastfeeding practices on postnatal HIV-1 transmission in Zimbabwe. Am J Public Health 2007; 97:1249-1254.

13. van Empelen $P$, Kok G, van Kesteren NM, van den Borne B, Bos $\mathrm{AE}$, Schaalma HP. Effective methods to change sex-risk among drug users: a review of psychosocial interventions. Soc Sci Med 2003; 57:1593-1608.

14. Berkman A, Garcia J, Muñoz-Laboy M, Paiva V, Parker R A critical analysis of the Brazilian response to HIV/AIDS: lessons learned for controlling and mitigating the epidemic in developing countries. Am J Public Health 2005; 95:1162-1172.

15. Abrams EJ, Myer L, Rosenfield A, El-Sadr WM. Prevention of mother-to-child transmission services as a gateway to familybased HIV care and treatment in resource-limited settings: rationale and international experiences. Am J Obstet Gynecol 2007; 197 (3 Suppl):S101-106.

16. Weinhardt LS, Kelly JA, Brondino MJ, Rotheram-Borus MJ, Kirshenbaum S, Chesney M, et al. HIV transmission risk behavior among men and women living with HIV in four US cities. J Acquir Immune Defic Syndr 2004; 36:1057-1066.

17. Marks G, Crepaz N, Senterfitt JW, Janssen RS. Meta-analysis of high-risk sexual behavior in persons aware and unaware they are infected with HIV in the United States: implications for HIV prevention programs. J Acquir Immune Defic Syndr 2005; 39:446-453.

18. Sweat M, Gregorich S, Sangiwa G, Furlonge C, Balmer D, Kamenga C, et al. Cost-effectiveness of voluntary HIV-1 counselling and testing in reducing sexual transmission of HIV-1 in Kenya and Tanzania. Lancet 2000; 356:113-121.

19. Denison JA, O'Reilly KR, Schmid GP, Kennedy CE, Sweat MD. HIV voluntary counseling and testing and behavioral risk reduction in developing countries: a meta-analysis, 19902005. AIDS Behav Epub ahead of print. 27 December 2007.

20. Richman DD, Morton SC, Wrin T, Hellmann N, Berry S, Shapiro $\mathrm{MF}$, et al. The prevalence of antiretroviral drug resistance in the United States. AIDS 2004; 18:1393-1401.

21. Blower SM, Aschenbach AN, Kahn JO. Predicting the transmission of drug-resistant HIV: comparing theory with data. Lancet Infect Dis 2003; 3:10-11.

22. Remien RH, Exner TM, Morin SF, Ehrhardt AE, Johnson MO, Correale J, et al. Medication adherence and sexual risk behavior among HIV-infected adults: implications for transmission of resistant virus. AIDS Behav 2007; 11:663-675.

23. Ostrow DE, Fox KJ, Chmiel JS, Silvestre A, Visscher BR, Vanable $\mathrm{PA}$, et al. Attitudes towards highly active antiretroviral therapy are associated with sexual risk taking among HIV-infected and uninfected homosexual men. AIDS 2002; 16:775-780.

24. Remien RH, Halkitis PN, O'Leary A, Wolitski RJ, Gomez CA. Risk perception and sexual risk behaviors among HIV-positive men on antiretroviral therapy. AIDS Behav 2005; 9:167-176.

25. Kerrigan D, Bastos FI, Malta M, Carneiro-da-Cunha C, Pilotto $\mathrm{JH}$, Strathdee SA. The search for social validation and the sexual behavior of people living with HIV in Rio de Janeiro, Brazil: understanding the role of treatment optimism in context. Soc Sci Med 2006; 62:2386-2396. 
26. Crepaz N, Hart TA, Marks G. Highly active antiretroviral therapy and sexual risk behavior: a meta-analytic review. JAMA 2004; 292:224-236.

27. Klitzman RL, Kirshenbaum SB, Dodge B, Remien RH, Ehrhardt $\mathrm{AA}$, Johnson $\mathrm{MO}$, et al. Intricacies and inter-relationships between HIV disclosure and HAART: a qualitative study. AIDS Care 2004; 16:628-640.

28. Bobrova N, Alcorn R, Rhodes T, Rughnikov I, Neifeld E, Power $R$. Injection drug users' perceptions of drug treatment services and attitudes toward substitution therapy: a qualitative study in three Russian cities. I Subst Abuse Treat 2007; 33:373378.

29. Lurie $P$. When science and politics collide: the federal response to needle-exchange programs. Bull NY Acad Med 1995; 72 : 380-396.

30. Agoramoorthy G, Minna JH. India's homosexual discrimination and health consequences. Rev Saude Publica 2007; 41:657660.

31. Beyrer C. War in the blood: sex, politics and AIDS in Southeast Asia. 1st ed. London and New York: Zed Books; 1998.

32. Cooper D, Harries J, Myer L, Orner P, Bracken H. "Life is still going on": reproductive intentions among HIV-positive women and men in South Africa. Soc Sci Med 2007; 65: 274-283.

33. Myer L, Morroni C, Rebe K. Prevalence and determinants of fertility intentions of HIV-infected women and men receiving antiretroviral therapy in South Africa. AIDS Patient Care STDS 2007; 21:278-285.

34. Paiva V, Filipe EV, Santos N, Lima TN, Segurado A. The right to love: the desire for parenthood among men living with HIV. Reprod Health Matters 2003; 11:91-100.

35. Sweat MD, O'Reilly KR, Schmid GP, Denison J, de Zoysa I. Cost-effectiveness of nevirapine to prevent mother-to-child HIV transmission in eight African countries. AIDS 2004; 18:1661-1671.

36. Myer L, Rebe K, Morroni C. Missed opportunities to address reproductive health care needs of HIV-infected women attending antiretroviral therapy services in Cape Town, South Africa. Trop Med Int Health 2007; 12 (12):14841489.

37. Morin SF, Koester KA, Steward WT, Maiorana A, McLaughlin M, Myers JJ, et al. Missed opportunities: prevention with HIVinfected patients in clinical care settings. I Acquir Immune Defic Syndr 2004; 36:960-966.

38. Luna GC, Rotheram-Borus MJ. Youth living with HIV as peer leaders. Am / Commun Psychol 1999; 27:1-23.

39. Bing EG, Burnam MA, Longshore D, Fleishman JA, Sherbourne $\mathrm{CD}$, London AS, et al. Psychiatric disorders and drug use among human immunodeficiency virus-infected adults in the United States. Arch Gen Psychiatry 2004; 58:721-728.

40. Lyketsos CG, Hanson A, Fishman M, McHugh PR, Treisman G]. Screening for psychiatric morbidity in a medical outpatient clinic for HIV infection: the need for a psychiatric presence. Int I Psychiatry Med 1994; 24:103-113.

41. Chandra PS, Desai G, Ranjan S. HIV and psychiatric disorders. Indian I Med Res 2005; 121:451-467.

42. Leserman J. HIV disease progression: depression, stress, and possible mechanisms. Biol Psychiatry 2003; 54:295306.

43. Tucker JS, Burnam MA, Sherbourne CD, Kung FY, Gifford AL. Substance use and mental health correlates of non-adherence to antiretroviral medications in a sample of patients with human immunodeficiency virus infection. $\mathrm{Am} / \mathrm{Med}$ 2003; 114:573-580.

44. Ramrakha S, Caspi A, Dickson N, Moffitt TE, Paul C. Psychiatric disorders and risky sexual behavior in young adulthood: cross sectional study in birth cohort. BMJ 2000; 321:263266.

45. Ammassari A, Antinori A, Aloisi MA, Trotta MP, Murri R, Bartoli L, et al. Depressive symptoms, neurocognitive impairment, and adherence to highly active antiretroviral therapy among HIV-infected persons. Psychosomatics 2004; 45 (5): 394-402.

46. Kresina TF, Flexner CW, Sinclair J, Correia MA, Stapleton JT, Adeniyi-Jones $\mathrm{S}$, et al. Alcohol use and HIV pharmacotherapy. AIDS Res Hum Retroviruses 2002; 18:757-770.
47. Evans DL, Ten Have TR, Douglas SD, Gettes DR, Morrison M, Chiappini MS, et al. Association of depression with viral load, CD8 T lymphocytes, and natural killer cells in women with HIV infection. Am J Psychiatry 2002; 159:752-759.

48. Pence BW, Miller WC, Gaynes BN, Eron JJ Jr. Psychiatric illness and virologic response in patients initiating highly active antiretroviral therapy. I Acquir Immune Defic Syndr 2007; 44:159166.

49. Remis RS, Bruneau J, Hankins CA. Enough sterile syringes to prevent HIV transmission among injection drug users in Montreal? J Acquir Immune Defic Syndr Hum Retrovirol 1998; 18 (Suppl. 1):S57-S59.

50. Hathaway AD Tousaw KI. Harm reduction headway and continuing resistance: insights from safe injection in the city of Vancouver. Int J Drug Policy 2008; 19:11-16.

51. Purcell DW, Metsch LR, Latka M, Santibanez S, Gomez CA, Eldred $L$, et al. Interventions for seropositive injectors-research and evaluation: an integrated behavioral intervention with HIV-positive injection drug users to address medical care, adherence, and risk reduction. I Acquir Immune Defic Syndr 2004; 7 (Suppl. 2):S110-S118.

52. Altice FL, Mezger JA, Hodges J, Bruce RD, Marinovich A, Walton $M$, et al. Developing a directly administered antiretroviral therapy intervention for HIV-infected drug users: implications for program replication. Clin Infect Dis 2004; 38 (Suppl. 5):S376-S387.

53. Hacker MA, Kaida A, Hogg RS, Bastos FI. The first ten years: achievements and challenges of the Brazilian program of universal access to HIV/AIDS comprehensive management and care, 1996-2006. Cad Saude Publica 2007; 23 (Suppl. 3): S345-S359.

54. Bastos FI, Nunn A, Hacker MA, Malta M, Szwarcwald CL. AIDS in Brazil: the challenge and the response. In: Edited by Celentano DD, Beyrer C. Public health aspects of HIVIAIDS in developing countries: epidemiology, prevention and care. New York: Springer International; 2008.

55. Institute of Medicine. No time to lose: getting more from HIV prevention. Washington, DC: National Academy of Sciences; 2001.

56. Maluwa M, Aggleton P, Parker R. HIV and AIDS-related stigma, discrimination, and human rights: a critical overview. Health Hum Rights 2002; 6:1-18.

57. Kamb ML, Fishbein M, Douglas JM, Rhodes F, Rogers J, Bolan G, et al. Efficacy of risk-reduction counseling to prevent human immunodeficiency virus and sexually transmitted diseases: a randomized controlled trial. Project RESPECT Study Group. JAMA 1998; 280:1161-1167.

58. Rotheram-Borus MJ, Lee MB, Murphy DA, Futterman D, Duan $N$, Birnbaum JM, Lightfoot $M$, and the Teens Linked to Care Consortium. Efficacy of a preventive intervention for youths living with HIV. Am J Public Health 2001; 91:400-405.

59. Rotheram-Borus MJ, Swendeman D Comulada WS, Weiss RE, Lee $M$, Lightfoot $M$. Prevention for substance-using HIVpositive young people: telephone and in-person delivery. J Acquir Immune Defic Syndr 2004; 37 (Suppl. 2):S68-S77.

60. Janssen RS, Holtgrave DR, Valdiserri RO, Shepherd M, Gayle HD, De Cock KM. The serostatus approach to fighting the HIV epidemic: prevention strategies for infected individuals. $\mathrm{Am} /$ Public Health 2001; 91:1019-1024.

61. Center for Disease Control and Prevention. HIV prevention strategic plan through 2005. Atlanta: CDC; 2001.

62. Bartholomew LK, Gold RS, Parcel GS, Czyzewski DI, Sockrider MM, Fernandez M, et al. Watch, discover, think, act: evaluation of computer-assisted instruction to improve asthma self-management in inner-city children. Patient Educ Counsel 2000; 39:269-280.

63. Schinke SP, DeNoia J, Glassman JR. Computer-mediated intervention to prevent drug abuse and violence among high-risk youth. Addict Behav 2004; 29:225-229.

64. Knebel EA. Operations research results. Comparison of computer-based and standard training in the integrated management of childhood illness in Uganda. Bethesda, MD: QA Project, Center for Human Services; May 2002.

65. Brechin SG. Implementing a new training approach: pilot test of ModCal ${ }^{\mathbb{R}}$ in Zimbabwe 1997. Available at: http://www.jhpiego. org/resources/pubs/TR/tr9728sum.htm. Accessed: 26 April 2007. 